\title{
Trabajo social chileno y dictadura militar. Memoria profesional predictatorial. Participación social en salud, período $1960-1973^{1}$
}

\author{
Patricia Castañeda ${ }^{2}$ y Ana María Salamé ${ }^{3}$
}

\begin{abstract}
El artículo presenta los resultados de un proyecto de investigación interuniversitario formulado en torno al rescate de la memoria colectiva de Trabajo Social durante el período 1973-1990, en el contexto de la dictadura militar. El objetivo de investigación corresponde a la reconfiguración del período profesional predictatorial en el contexto de la participación comunitaria en salud y su influencia en la formación y desempeño profesional. La metodología de investigación es cualitativa, la estrategia de recolección de información es la entrevista biográfica. Los datos se analizan con relación al objetivo de investigación y su eje temporal. Los resultados son el análisis del período pre dictatorial 19601973 desde la dinámica de la participación social en salud; la contribución de Trabajo Social en dicho proceso y los efectos generados como resultado del golpe militar de 1973.
\end{abstract}

Palabras clave: Trabajo Social chileno, dictadura militar, memoria profesional, participación social en salud.

\section{Trabalho social chileno e ditadura militar. Memória profissional pré-ditatorial. Participação social em saúde, período 1960-1973.}

\section{RESUMO}

O artigo apresenta os resultados dum projeto de investigação interuniversitária formulada em torno ao resgate da memória coletiva de Trabalho Social durante o período 1973-1990, no contexto da ditadura militar. O objetivo de investigação corresponde à reconfiguração do período profissional préditatorial no âmbito da participação comunitária em saúde e sua influência

Artículo recibido: 01/05/2016. Artículo aprobado: 09/08/2016.

Chilena. Trabajadora social. Académica e investigadora Escuela de Trabajo Social, Universidad de Valparaíso. Correo electrónico: patricia.castaneda@uv.cl.

3 Chilena. Trabajadora social. Académica e investigadora Departamento de Trabajo Social, Universidad de La Frontera. Correo electrónico: ana.salame@ufrontera.cl 
na formação e desempenho profissional. A metodologia de investigação é qualitativa, a estratégia de coleta de informação é a entrevista biográfica. Os dados se analisam com referente ao objetivo de investigação e seu eixo temporal. Os resultados são a análise do período pré-ditatorial 1960-1973 a partir da dinâmica da participação social em saúde; a contribuição do Trabalho Social em aquele processo e os efeitos gerados como resultado do golpe militar de 1973.

Palavras-chave: trabalho social chileno, ditadura militar, memória profissional, participação social em saúde.

\section{Social work chilean and military dictatorship. Memory predictatorial professional. Social participation in health, 1960-1973 period}

\footnotetext{
ABSTRACT

This article presents the results of an inter-university research project designed around the rescue of the collective memory of Social Work during the period 1973-1990, in the context of the military dictatorship. The objective of research corresponds to the reconfiguration of the pre dictatorial professional period in the context of community participation in health and its influence on the formation and professional performance. The research methodology is qualitative, data collection strategy is the biographical interview. The data are analyzed with reference to the objective of research and its temporal axis. The results are the analysis of the pre dictatorial period from 1960-1973 from the dynamics of social participation in health; the contribution of social workers in the process and the effects generated as a result of the military coup of 1973.
}

Keywords: Chilean social work, military dictatorship, professional memory, social participation in health..

\section{Antecedentes generales}

A inicios de la década de 1960 y con poco más de 6 millones de habitantes, Chile enfrentaba múltiples problemas sanitarios, derivados de las condiciones de vida propias de la región de América Latina. Así entonces, se caracterizaba por ser un país afectado por el subdesarrollo económico, con preeminencia demográfica de la población joven y un acelerado proceso de urbanización en plena conformación. La edad promedio de la población era de 26 años, con sólo 19 adultos mayores por cada cien menores 
de 15 años. El 68,2\% de la población vivía en zonas urbanas y el $31,8 \%$ en zonas rurales. Los indicadores sanitarios informaban que la cobertura de atención profesional del parto era de $66,9 \%$; la tasa de mortalidad infantil de 117,1 por mil nacidos vivos; y la tasa de mortalidad materna alcanzaba 118 muertes maternas por 100 mil nacidos vivos. La principal causa de muerte materna correspondía a abortos provocados, ante la ausencia de programas de planificación familiar en los sistemas de salud pública. La tasa global de fecundidad correspondía a 5,4 hijos por mujer y la esperanza de vida al nacer era en promedio 57,1 años para ambos sexos. Las principales causas de mortalidad correspondían a sistema circulatorio (21\%), afecciones perinatales (14\%) y enfermedades del sistema digestivo (12\%). La desnutrición infantil alcanzaba el 37\% en menores de 6 años. La mayoría de la población carecía de servicios básicos y saneamiento ambiental, por lo que se presentaba una alta prevalencia de diarreas, bronconeumonías y enfermedades infecciosas en la población infantil, dadas las condiciones de pobreza en las que se encontraban sus familias. El promedio de años de escolaridad aprobados por la población de 15 años o más correspondía a 4,9 y el 16,9\% de la población no contaba con estudios básicos o era analfabeta (INE, 1960; Villalón y Vera, 2012: 37-63).

En este marco de acción, las políticas sanitarias chilenas se canalizaron centralmente a través del Servicio Nacional de Salud (S.N.S.), repartición gubernamental de carácter centralizado creada en 1952, responsable de implementar las acciones de salud pública en el país. Su atención abarcó transversalmente a toda la población, con especial énfasis en los sectores de menores ingresos. Se impulsaron servicios gratuitos de medicina preventiva, política nacional de planificación familiar, control pre y post natal, atención hospitalaria del parto, estimulación de la lactancia materna y control de niño sano con cobertura de vacunaciones y alimentación complementaria. Además, el sistema de salud amplió progresivamente su cobertura a todo el país, generando una red de salud integrada y de complejidad creciente (postas rurales, centros de 
atención primaria y hospitales) que permitió extender la acción sanitaria a toda la población (Goic, 2015: 778).

En la década de 1960, durante el gobierno de Eduardo Frei Montalva (1964-1970), se respaldó la política de enviar médicos generales de zona a los sectores apartados del país, iniciativa originada en 1955, permitiendo constituir equipos de salud que otorgaron amplia cobertura de atención, con especial énfasis en la disminución de la mortalidad materna e infantil. A su vez, se crearon consultorios periféricos en sectores urbanos, requeridos como respuesta sanitaria a la masiva migración campo/ciudad que caracterizó los procesos de urbanización de las décadas de 1950 y 1960 en el país y que sobrepasó la infraestructura urbana de salud disponible. Todas estas acciones fueron apoyadas por una importante participación de la comunidad, mediante la creación de Consejos Comunales de Salud y Consejos Paritarios en consultorios y hospitales, acciones respaldadas a través del Decreto No 250 de 1967 del Ministerio de Salud, permitiendo que la población contribuyera activamente al desarrollo y consolidación de las iniciativas.

En julio de 1970 se celebró en Santiago de Chile la Primera Conferencia Internacional sobre Desarrollo de la Comunidad, donde se postuló como eje fundamental la incorporación de los sectores populares, mediante su participación organizada en fases de definición y acción en torno a los problemas que les aquejaban. Bajo estos postulados, se elabora en el Gobierno de Salvador Allende Gossens (1970-1973) un marco legal para la constitución de Consejos Locales y Consejos Paritarios de Salud a nivel de establecimientos (consultorios de atención primaria y hospitales) y Áreas de Salud en todo el Servicio Nacional de Salud, junto con medidas tendientes a aumentar la cobertura de la atención primaria y fortalecer las medidas sanitarias ya iniciadas en el gobierno anterior.

Durante el referido período 1960-1973, Trabajo Social -como integrante histórico de los equipos de salud- aportó su saber profesional en torno al nuevo desafío que representó integrar la participación social a las acciones sanitarias. Su desempeño asistencial tradicio- 
nal se vio resignificado, permitiéndole aportar de manera destacada en la convocatoria de la participación social que buscaba enfrentar propositivamente los desafíos por los que atravesaba el país en materia de salud. En este marco, el presente artículo tiene por objetivo reconstruir la memoria colectiva profesional de Trabajo Social en torno a la participación comunitaria en salud en el período predictatorial, valorizando las principales características que asumen la formación y el ejercicio profesional en dicho período.

\section{Metodología}

El presente artículo es resultado de una investigación interuniversitaria que busca rescatar y valorizar la memoria colectiva del Trabajo Social chileno en el contexto de la dictadura militar y de sus años precedentes (Castañeda y Salamé, 2013). El objetivo específico analizado en esta oportunidad, corresponde a la reconfiguración del período profesional pre dictatorial en el contexto de la participación comunitaria en salud y su influencia en la formación y desempeño profesional. Considerando que el objetivo se orienta a reconstruir la memoria de Trabajo Social, la metodología utilizada es de carácter cualitativo.

Los sujetos participantes en la investigación son 35 trabajadores y trabajadoras sociales en total, de los cuales 16 se desempeñaban como profesionales en la época en estudio y 19 como estudiantes. Los guiones de entrevista fueron diferenciados conforme el rol declarado por cada sujeto. La estrategia de recolección de información corresponde a entrevistas biográficas. Los sujetos participantes fueron contactados inicialmente a través de redes profesionales de trabajadores y trabajadoras sociales de salud en ejercicio y en situación de jubilación, junto a contactos proporcionados por el Colegio Profesional. Posteriormente, los contactos se realizaron a través del procedimiento de snowball o bola de nieve.

La recolección de información se realizó entre los meses de abril a octubre del año 2015. La información recogida en entrevistas se complementó con la revisión documental de archivos de las escuelas universitarias tradicionales, cuerpos legales vinculados 
a la profesión y publicaciones profesionales de la época. La revisión documental aportó información relevante de los contextos en los cuales se desarrollaron las experiencias relatadas. Con fines analíticos, la información se organizó cronológicamente con relación a los períodos presidenciales que enmarcan el estudio. De esta forma, la memoria profesional se presenta estructurada temporalmente, rescatando los énfasis específicos de cada período de estudio. Se mantiene el lenguaje sexista sin correcciones, referido directamente al contexto y a los documentos de la época.

\section{Resultados}

\section{Participación Comunitaria en Salud en el período de gobierno de Eduardo Frei Montalva 1964-1970}

En la década de 1960, el Estado de Chile orientó sus procesos de desarrollo social a partir de dos estrategias centrales: aumento de la cobertura educacional e impulso de la participación social de la población. Ambas estrategias se sintetizaron durante el gobierno demócrata cristiano de Eduardo Frei Montalva (1964-1970) en el concepto de Promoción Popular. Este concepto representaba "la incorporación del hombre común a través de los organismos que él integre y lo representen (...) a la posibilidad de expresarse, influir, opinar, exigir cuando sea justo y de contribuir de una manera organizada a las transformaciones que el país espera y requiere" (Frei, 1964: 19). Así entonces, se definía la Promoción Popular como un proceso que permitía "reconocer y promover las instituciones; crear y otorgar los servicios y recursos -cuando sea necesario- para que el pueblo se organice y asuma el papel que le corresponde en una sociedad moderna y significa también el acondicionamiento de las estructuras y mecanismos del Estado que permita a este mismo pueblo organizado incorporarse eficazmente al ejercicio del poder" (Frei, 1964: 20).

Estas declaraciones tuvieron implicancias concretas en cada ámbito de acción del trabajo ministerial del país, correspondiéndole al Ministerio de Salud proponer sus lineamientos a través del Ser- 
vicio Nacional de Salud, institucionalidad de la época responsable de implementar las acciones de salud pública en el país. Su acción tuvo como principal fundamento el "contacto permanente y directo que mantiene el SNS con la comunidad a través de sus diferentes funcionarios, que lo colocan en una situación de privilegio para conocer las necesidades sentidas de la comunidad" (SNS, 1962: 23). La propuesta de sumar la participación social al sector salud se inició con programas de trabajo directo en terreno, que tuvieron como objetivos "inventariar las organizaciones populares existentes y asesorarlas; promover la formación de otras, según las aspiraciones y necesidades de la comunidad y establecer planes concretos de acción comunitaria" (Frei, 1964: 24).

En este nuevo marco de acción, Trabajo Social fue redefiniendo su repertorio profesional y enriqueciéndolo con nuevas funciones y tareas que respondían a los desafíos que surgían desde las medidas gubernamentales. A la fecha, sus funciones tradicionales en los equipos de salud estaban asociadas, principalmente, a la asistencialidad: "las asistentes sociales enfrentan (...) un desafío más difícil. Sus funciones se refieren a aspectos menos instrumentales, menos "visibles" del cuidado de la salud y los resultados que logran están condicionados por el régimen mismo que engendra la miseria. Sólo aquellos equipos que trabajan con la doctrina de la medicina integral ${ }^{4}$ no tienen dificultad en valorar el aporte del servicio social" (Montoya y Videla, 1967: 16). Dado que el proceso de desarrollo profesional se encontraba estrechamente vinculado al sector salud, la profesión se adscribió rápidamente a las nuevas oportunidades que representaba la incorporación de la dimensión

Medicina Integral: Concepción de la medicina que considera al ser humano en una triple dimensión física, psíquica y social, en interacción con su medio ambiente. Actúa con una concepción total y ecológica del ser humano y del fenómeno saludenfermedad, y considera acciones integradas de salud, de Fomento, Recuperación y Rehabilitación de la salud, realizadas por un equipo de salud. Devoto (1970). Artículo: La medicina comunitaria y las vías de desarrollo socioeconómico. En: Cuadernos Médico Sociales 3, 3-9. Santiago de Chile: Colegio Médico de Chile. Recuperado el 25.11.2016 en: http://cms.colegiomedico.cl/Magazine/1970/11/3/11_3_9.pdf. 
comunitaria en su quehacer y reconoció en la participación social su estrategia central de trabajo sanitario.

A fin de respaldar el desempeño profesional en torno a la nueva estrategia promocional, en 1962 es invitada a Chile la Dra. Caroline Ware, asesora técnica de la Organización de Estados Americanos, funcionaria de la Oficina Panamericana de la Salud y autora de las obras clásicas Estudio de la Comunidad (1952) y Organización de la Comunidad para el Bienestar Social (1954). Durante su permanencia en el país realiza acciones formativas en trabajo comunitario con profesionales del Servicio Nacional de Salud y con equipos docentes de las escuelas de Servicio Social Dr. Alejandro del Río y Dr. Lucio Córdova. Asimismo, asesora en el diseño de los primeros programas de intervención comunitaria en salud, destinados a educación sanitaria y saneamiento ambiental en extensión de redes de agua potable y alcantarillado en las principales ciudades del país (López, 2014: 92-93). Consecuentemente, los equipos de Trabajo Social de Salud comenzaron a poner en práctica estrategias de intervención social, orientando sus acciones profesionales "a mejorar los problemas de salubridad, saneamiento ambiental y equipamiento comunitario, con el apoyo técnico y económico de organismos nacionales e instituciones internacionales. Junto con participar en procesos de erradicación de poblaciones "callampas", las asistentes sociales habilitaron escuelas, guarderías y jardines infantiles y formaron centros de madres y clubes juveniles" (Figueroa, 1976: 120).

Así, entonces, las profesionales que se desempeñaban en el Servicio Nacional de Salud iniciaron experiencias basándose en el método de Desarrollo de la Comunidad. Las experiencias piloto fueron implementadas en el Centro de Asistencia Médico Social San Joaquín; en el Departamento de San Felipe; en los sectores de Santa Inés y Forestal en Viña del Mar y Porvenir Alto y Cerro Las Cañas en Valparaíso. Los aprendizajes obtenidos orientaron las acciones sanitarias comunitarias que se impulsarían progresivamente a lo largo del período (Gutiérrez y Villamar, 1966: 38). A continuación, se ilustra una de las experiencias pioneras desarrolladas en el tema. 
Tabla No1. Experiencia profesional piloto salud comunitaria

\begin{tabular}{|c|c|}
\hline Nombre experiencia & Experiencia piloto de Desarrollo Comunitario Santa Inés \\
\hline Institución & $\begin{array}{l}\text { Servicio Nacional de Salud. Programa de Desarrollo Co- } \\
\text { munitario. Área Hospitalaria Viña del Mar. Sector Santa } \\
\text { Inés. }\end{array}$ \\
\hline Equipo de salud & $\begin{array}{l}\text { Equipo asesor: Asistente Social Jefe, Enfermera Subjefe, } \\
\text { Médico Asesor del programa y Educadora Sanitaria. } \\
\text { Equipo de Terreno: } 2 \text { Asistentes Sociales, } 1 \text { Enfermera de } \\
\text { Salud Pública, } 1 \text { Inspector Sanitario y } 1 \text { Educador Sani- } \\
\text { tario. }\end{array}$ \\
\hline Período & 1961 \\
\hline & Desc \\
\hline \multirow{3}{*}{\multicolumn{2}{|c|}{$\begin{array}{l}\text { Campaña informativa de inicio del Programa de Desarrollo Comunitario en el } \\
\text { sector. } \\
\text { Realización de Catastro de Organizaciones comunitarias existentes. } \\
\text { Aplicación de encuesta antecedentes socioeconómicos, saneamiento ambiental y } \\
\text { condiciones habitacionales. }\end{array}$}} \\
\hline & \\
\hline & \\
\hline \multicolumn{2}{|c|}{$\begin{array}{l}\text { Constitución de Comité Coordinador constituido por } 15 \text { organizaciones: Juntas } \\
\text { de Vecinos, Cooperativas, Centro Cultural, Clubes Deportivos, Instituciones Mu- } \\
\text { tualistas e Iglesias. }\end{array}$} \\
\hline \multicolumn{2}{|c|}{$\begin{array}{l}\text { Diagnóstico de problemas comunitarios: Se priorizan con participación de la co- } \\
\text { munidad Urbanización (sectores sin agua potable ni alcantarillado, desagües, pa- } \\
\text { vimentación, arreglo de calles, alumbrado público, basurales, muros de conten- } \\
\text { ción) y Alcoholismo. }\end{array}$} \\
\hline \multicolumn{2}{|l|}{ Acciones realizadas: } \\
\hline \multicolumn{2}{|c|}{ 1. Curso de capacitación de líderes comunitarios. } \\
\hline \multicolumn{2}{|c|}{$\begin{array}{l}\text { 2. Campaña de Saneamiento Ambiental. Ejecutada por organizaciones comunita- } \\
\text { rias, Servicio Nacional de Salud y Municipalidad. }\end{array}$} \\
\hline \multicolumn{2}{|c|}{$\begin{array}{l}\text { 3. Prevención en Alcoholismo. Desarrollo de Unidad de Enseñanza en la Escuela } \\
\text { Pública de una semana de duración. Asamblea de Centro de Padres. Exposición } \\
\text { de trabajos confeccionados por los alumnos. }\end{array}$} \\
\hline \multicolumn{2}{|c|}{$\begin{array}{l}\text { 4. Intervención Educativa en Alcoholismo. Programa de Higiene Mental a cargo } \\
\text { de médico psiquiatra, asistente social y enfermeras del Servicio de Neuropsi- } \\
\text { quiatría del Hospital de Viña del Mar. }\end{array}$} \\
\hline \multicolumn{2}{|c|}{$\begin{array}{l}\text { 5. Coordinación con Municipalidad de Viña del Mar para limitar número de pa- } \\
\text { tentes de venta de alcoholes en el sector. }\end{array}$} \\
\hline
\end{tabular}


Juicios Evaluativos de la Experiencia Piloto

“Todas las acciones realizadas se lograron a través de comisiones de trabajo en las que participaron los técnicos y la población, mediante una integración de las instituciones en un Comité Coordinador y a través del nacimiento de otras instituciones.

Se aprecia que el programa en general se abocó a la solución de problemas de tipo material, por constituir éstos los más sentidos por la población, observándose que no están conscientes de los problemas de índole espiritual (recreación, cultura y otros).

En la planificación se consideraron formas de lograr la sensibilización y participación activa de la población, tales como asambleas, reuniones, visitas domiciliarias, entrevistas y medios de difusión. Igualmente, en consideración a que el líder es un factor importante para lograr los objetivos señalados, se planificó la capacitación de líderes como una necesidad sentida por los técnicos.

Respecto a la falta de preparación de los líderes de la comunidad, el equipo técnico tuvo conciencia de ello y los dirigentes también están conscientes que es necesario adquirir mayor experiencia y capacitar nuevos líderes".

Fuente Experiencia Profesional Piloto: Gutiérrez A. y Villamar K. (1966). Ver detalles en bibliografía.

Con fecha 15 de mayo de 1967, se promulgó el Decreto $\mathrm{N}^{\circ} 250$ del Ministerio de Salud, que creó los Consejos Comunitarios de Salud, formalizando la incorporación de representantes de organizaciones vecinales territoriales y funcionales en acciones de asesoría o consulta de los problemas y necesidades de salud de la población. El cuerpo legal establecía que los Consejos Comunitarios podían "examinar los problemas de salud que afecten a la comunidad; propender a que ellos sean solucionados mediante acciones rápidas y eficaces; promover el interés de los habitantes para participar en forma activa en la solución de los mismos; colaborar en la divulgación de los planes y acciones de salud que programe la autoridad; representar las anomalías que aparezcan en la ejecución de esas acciones y, en general, procurar un mayor acercamiento de la comunidad con los establecimientos que ejecutan acciones de salud" (Art. 3). A juicio de los equipos profesionales de salud, esta nueva concepción del sentido y forma de las acciones sanitarias "debe transformarse en un ideal, con 
un dinamismo tal que permita al individuo y a la comunidad sentirse sujeto activo en este proceso de cambio y no un objeto pasivo que recibe beneficios de un grupo que decide las acciones sin una participación comunitaria efectiva y solidaria" (Ugarte, 1970: 32).

Estas nuevas orientaciones se reflejan en las experiencias formativas de Trabajo Social, que comienzan a transitar desde una tradición histórica en el sector salud caracterizada por una impronta marcadamente asistencial, hacia las nuevas oportunidades derivadas de la incorporación activa de la participación comunitaria en las preocupaciones sanitarias. A continuación se ilustra una experiencia de práctica profesional en el nuevo marco de la promoción social.

Tabla No2. Práctica profesional trabajo comunitario en salud

\begin{tabular}{|l|l|}
\hline Nombre experiencia & Proyecto de Acción Comunitaria de Quinta Normal \\
\hline Instituciones & $\begin{array}{l}\text { Escuela de Servicio Social, Universidad de Chile, sede } \\
\text { Santiago, y Servicio Nacional de Salud. Área Occidente. }\end{array}$ \\
\hline Equipo de trabajo & $\begin{array}{l}13 \text { estudiantes en Práctica de Comunidad, Asistente } \\
\text { Social Docente de Cátedra, Asistente Social Supervi- } \\
\text { sora Institucional, Educador Sanitario del Área, Inge- } \\
\text { niero Jefe de la Oficina de Higiene Ambiental del Ârea, } \\
\text { Enfermera Jefe y Médico Director }\end{array}$ \\
\hline Período de trabajo & 1966 \\
\hline \multicolumn{1}{|c|}{ Propósitos } \\
\hline $\begin{array}{l}\text { "i) Establecer comunicación y coordinación entre el Servicio Nacional de Salud } \\
\text { y la población a través de las organizaciones, con el objeto de elevar el nivel de } \\
\text { salud de sus integrantes; ii) Promover en las organizaciones de base la búsqueda } \\
\text { adecuada de soluciones a las necesidades más sentidas de la población; y, iii) Pro- } \\
\text { mover la participación de los técnicos y de las instituciones para el mejor desa- } \\
\text { rrollo del programa en relación con salud y para un mejor aprovechamiento de } \\
\text { los recursos existentes". }\end{array}$ \\
\hline \multicolumn{1}{|c|}{ Objetivo Docente } \\
\hline $\begin{array}{l}\text { "Entrenar a los alumnos en la aplicación y manejo del Método de Organización y } \\
\text { Desarrollo de la Comunidad a través de un programa elaborado a nivel del Ser- } \\
\text { vicio Nacional de Salud". }\end{array}$ \\
\hline
\end{tabular}




\section{Descripción de la Experiencia}

Intervención directa en el Consultorio Andes que incluyó 8 sectores y 40 organizaciones que recibieron asesoría social directa en temáticas de manejo de acequias y canales, extracción de basuras, problemas de higiene ambiental y falta de equipamiento del Hospital Félix Bulnes.

Jornadas Comunitarias con contenidos educativos definidos por la propia población y que correspondieron a: Servicio de Seguro Social, Servicio Nacional de Salud, Organización y Desarrollo de la Comunidad, Líder y Liderazgo y Relaciones Humanas. Motivación y organización de charlas sobre métodos y uso de anticonceptivos, dadas a nivel de organizaciones de base. Análisis de los contenidos con los grupos de la comunidad.

Elevar los índices de vacunación de antivariólica, BCG, sarampión, polio, mixta y difteria a través de charlas, proyección de película, reparto de cartillas, foro, motivación en las industrias y otros grupos comunitarios y encuestaje de 840 familias con los miembros de la comunidad.

Coordinación del trabajo de equipo de comunidad con el trabajo de madres líderes. Trabajo de Investigación sobre características de los problemas de salud.

Fuente: Universidad de Chile (1966). Ver detalles en bibliografía.

En un escenario de profundos cambios estructurales, la política social giraba abiertamente hacia la declaración de promoción popular vigente. Para el colectivo profesional que se incorporaba a la acción sanitaria en el marco comunitario, la experiencia representaba una oportunidad de aprendizaje que respaldaba una empleabilidad favorable: "Me acuerdo que mi primera práctica la hice en un centro materno infantil, donde se estaba iniciando el tema comunitario. Había que trabajar con la comunidad. Era interesante como experiencia porque los programas estaban recién partiendo, no existía mucha información al respecto y uno tenía que tirarse al agua y aprender con la misma gente. Había muy pocas asistentes sociales en el tema comunitario, así que rápidamente contrataban a todas las niñas y pagaban harto" (Estudiante Trabajo Social. Período 1967-1971).

Otro testimonio similar relata: "Yo me di el lujo de no trabajar al tiro cuando me titulé. Acompañé a una colega a buscar trabajo en el Servicio Nacional de Salud y me dijeron: ¿Ud. se interesaría en trabajar? Y yo dije bueno, pero en enero. Y estábamos en septiembre. Así que me esperaron de septiembre a enero para que empezara a trabajar en salud" (Trabajadora Social de Salud. Año de Ingreso al Servicio, 1968). 
Con fecha 19 de julio de 1968 se promulga la Ley $\mathrm{N}^{\circ} 16.880$ de Organizaciones Comunitarias, que formaliza legalmente la participación social al distinguir dos tipos de organizaciones comunitarias: territoriales y funcionales. Asimismo, reconoce a las Juntas de Vecinos como "una expresión de solidaridad y organización en el ámbito territorial para la defensa permanente de los asociados y como colaboradoras de la autoridad del Estado y de las municipalidades" (Art. 1). Con el nombre de organizaciones funcionales, la ley reconoce también a otras organizaciones comunitarias, tales como "centros de madres, centros de padres y apoderados, centros culturales y artísticos, organizaciones juveniles, organizaciones deportivas, grupos corales, cooperativas y otras que tengan carácter similar, que representen y promuevan valores específicos de la comunidad vecinal" (Art. 1). La ley reconoce a todas las organizaciones comunitarias "el derecho a constituir organismos que las representen en los distintos niveles de la vida nacional, en Agrupaciones, Uniones, Federaciones y Confederaciones" (Art. 1). Las medidas impulsaron los procesos de participación social a través de la formación de organizaciones comunitarias a lo largo del país. A la profesión le correspondió acompañar dichos esfuerzos y traducir la política social en acciones promocionales de carácter educativo sanitario: "En ese tiempo, las organizaciones comunitarias eran más estructuradas. La gente pertenecía a los organismos básicos de la comunidad, principalmente a las juntas de vecinos y los centros de madres. El trabajo con las organizaciones permitía conocer su historia, sus mitos, sus creencias y así se generaban confianzas entre el equipo de salud y los pobladores para trabajar en forma organizada" (Estudiante Trabajo Social. Período 1967-1971).

Los aprendizajes profesionales de Trabajo Social respecto de la participación social en el contexto sanitario, permitía constatar que los problemas y necesidades más apremiantes de la población no podían ser superados exclusivamente desde esfuerzos individuales. Por tanto, el esfuerzo colectivo ofrecía nuevas perspectivas de solución a grupos sociales territorialmente definidos: "Antiguamente, la asistente social era la responsable de hacer desarro- 
llo de la comunidad, trabajaba con las organizaciones, ya fueran funcionales o territoriales. La asistente social se conocía la vida de la comunidad. Se juntaban los dirigentes y se organizaban actividades que fueran en beneficio de la misma población" (Trabajadora Social de Salud. Año de Ingreso al Servicio, 1970).

En este contexto de impulso a la participación social, la profesión incorpora estrategias de trabajo comunitario en el tratamiento y rehabilitación de las problemáticas de salud de la época, las que tradicionalmente habían sido enfrentadas con tratamientos eminentemente clínicos. A continuación se ilustra una experiencia que detalla las estrategias referidas.

Tabla No3. Experiencia de participación comunitaria en rehabilitación

\begin{tabular}{|l|l|}
\hline Nombre experiencia & Club de Abstemios \\
\hline Institución & Hospital de Coronel \\
\hline Equipo de salud & $\begin{array}{l}\text { Médico, Enfermera, Matrona, Asistente Social, } 4 \text { Auxilia- } \\
\text { res de Enfermería y 2 Oficiales Administrativos. }\end{array}$ \\
\hline Período de trabajo & 1967-1970 \\
\hline \multicolumn{1}{|c|}{ Descripción de la Experiencia } \\
\hline $\begin{array}{l}\text { "Nos propusimos con la asistente social trabajar con alcohólicos, naciendo nuestro } \\
\text { club de abstemios. Pero nuestra filosofía era aprovechar todo lo que se hiciera y no } \\
\text { se perdiera como sucede habitualmente: el médico envía su paciente a un centro es- } \\
\text { pecializado y luego regresa sin que nadie lo reciba ni lo siga orientando, perdiendo } \\
\text { recursos el Servicio Nacional de Salud al volver el paciente a la bebida (...) Nosotros } \\
\text { operamos del siguiente modo: el paciente alcohólico que desea hacerse tratamiento } \\
\text { pasa a la asistente social, quien lo encuesta y conversa con él; luego me lo envía y se } \\
\text { hospitaliza para "desintoxicarlo" en un par de semanas (diez a catorce días) y luego } \\
\text { tenemos una larga entrevista. Veo la posibilidad de enviarlo con sus datos clínicos, de } \\
\text { laboratorio y con su evolución a un especialista; este después me lo devuelve y yo sigo } \\
\text { controlándolo. Mientras la asistente social va a la casa y prepara el terreno para cuan- } \\
\text { do vuelva el paciente. Ingresa al club, el cual se reúne semanalmente y con sus mu- } \\
\text { jeres mensualmente. (...) Para navidad se organizó un trabajo en el mes de noviem- } \\
\text { bre, de arreglos pascueros y así, en una exposición mostraron sus trabajos ganando } \\
\text { premios. Por primera vez en sus vidas, muchos de estos mineros supieron lo que } \\
\text { era una pascua y aún más, la pasaron sobriamente. Han pasado por este sistema de } \\
\text { acción antialcohólica 50 pacientes, } 46 \text { hombres y } 4 \text { mujeres y con el control periódico } \\
\text { del médico y la asistente social hemos logrado buenos resultados". }\end{array}$ \\
\hline \begin{tabular}{l} 
Fuente: Sotomayor H. y cols. (1971). Ver detalles en bibliografía. \\
\hline
\end{tabular}
\end{tabular}


La participación comunitaria en salud podía expresarse a través de tres mecanismos. El primero poseía un carácter directo, a través de la incorporación de representantes de las organizaciones territoriales y funcionales en los Consejos Comunitarios de Salud. El segundo mecanismo correspondía a la ayuda técnica, financiera o de recursos que podían dar los organismos de salud a instituciones creadas y mantenidas por la comunidad para realizar acciones de salud. El tercer mecanismo era la ayuda directa de los organismos privados a las instituciones de salud, para la ejecución de acciones o para realizar obras que significaran un nivel de mejoramiento del nivel de salud de la población (Ugarte, 1970: 32). A continuación se ilustra una experiencia de participación comunitaria a través del segundo y tercer mecanismo en forma integrada.

Tabla No 4 . Experiencia de participación comunitaria en mejoramiento de infraestructura y servicio sanitarios

\begin{tabular}{|c|c|}
\hline Nombre experiencia & $\begin{array}{l}\text { Modelo de acción cooperativa para mejoramiento del } \\
\text { nivel de salud de la población }\end{array}$ \\
\hline Institución & Hospital de Vallenar \\
\hline Equipo de salud & $\begin{array}{l}15 \text { Médicos, } 5 \text { Enfermeras, } 2 \text { Dentistas, } 5 \text { Matronas, } 1 \\
\text { Asistente Social, } 72 \text { Auxiliares de Enfermería, } 2 \text { Auxilia- } \\
\text { res Sociales, } 17 \text { Funcionarios Administrativos, } 49 \text { Perso- } \\
\text { nal de Servicio y } 6 \text { Choferes. }\end{array}$ \\
\hline Período de trabajo & 1964-1968 \\
\hline \multirow{2}{*}{\multicolumn{2}{|c|}{$\begin{array}{l}\text { "En este momento se está terminando de construir un cuarto consultorio perifé- } \\
\text { rico en Población Gómez, de corte más moderno y amplio, cuya ejecución (...) es } \\
\text { con aportes en trabajo y en dinero de los propios pobladores, donación del sitio y } \\
\text { aporte en materiales de la Municipalidad de Vallenar, donación de la obra gruesa } \\
\text { y dirección técnica a cargo del Rotary Club e integración de miembros del equipo } \\
\text { de salud al comité ejecutivo que dirige esta obra, asesorando en múltiples formas } \\
\text { (...) Los pobladores de Junta del Carmen hace sólo algunos meses entregaron una } \\
\text { excelente posta rural, en un esfuerzo digno de elogio. El Servicio hizo aportes de } \\
\text { importancia tanto material como técnica, en este trabajo cooperativo. En igual for- } \\
\text { ma, por esfuerzo de sus pobladores, se está terminando de ampliar la posta rural en } \\
\text { La Laja y, en sus últimos detalles, la de Chañar Blanco". }\end{array}$}} \\
\hline & \\
\hline Fuente: Puentes, R. (196 & Ver detalles en bibliografía. \\
\hline
\end{tabular}


La formación profesional acoge favorablemente las experiencias de participación social en salud. Las escuelas de Trabajo Social dependientes de las sedes de la Universidad de Chile incorporan formalmente en el plan de estudios la asignatura de Organización y Desarrollo de la Comunidad en el año 1967, aunque con anterioridad a esta declaración formal ya se realizaban pasantías y experiencias formativas en contextos comunitarios. Asimismo, se realizan experiencias de titulación, como lo demuestran las siguientes tesis revisadas en la sede Valparaíso de la Universidad de Chile: "Aspectos que favorecen o dificultan un programa de organización y desarrollo de la comunidad en un área urbana" (1966); "Rol del asistente social en un equipo de salud pública en un programa de saneamiento básico con comunidades con otros servicios" (1967); "Los centros de madres y su proyección en la mujer, la familia y la comunidad" (1968); "Plan de desarrollo comunitario para la localidad de Valle Hermoso, La Ligua” (1969); “Una experiencia de aplicación del método de desarrollo y organización de la comunidad en un programa de erradicación" (1969); y, "La organización vecinal como medio de participación para el cambio" (1969).

Los esfuerzos por impulsar la participación comunitaria en los procesos sanitarios realizados por los equipos de salud en general y por Trabajo Social en particular, tuvieron un alcance total estimado en dos millones de personas, que para el año 1970 se encontraban participando del proceso de toma de decisiones en el nivel intermedio, con cerca de 20.000 organizaciones de base constituidas formalmente (Lavados, 1983). Con ello se superó ampliamente la meta propuesta por el gobierno demócrata cristiano que esperaba extender la acción organizada a 600.000 personas al final de su período (Frei, 1964).

\section{Participación comunitaria en Salud en el período de gobierno de Salvador Allende Gossens, 1970- 1973}

El gobierno de Salvador Allende profundizó los procesos en torno a la participación, a través de una redefinición de mayor compromiso social que fue representada por medio del concepto de Poder 
Popular. Así entonces, el Programa de Gobierno de la coalición de las fuerzas de izquierda agrupadas en la Unidad Popular proponía la generación de un sujeto popular revolucionario a través de la concientización, dado que "las transformaciones revolucionarias que el país necesita solo podrán realizarse si el pueblo chileno toma en sus manos el poder y lo ejerce real y efectivamente, (...) las organizaciones sindicales y sociales de los obreros, empleados, campesinos, pobladores, dueñas de casa, estudiantes, profesionales, intelectuales, artesanos, pequeños y medianos empresarios y demás sectores de trabajadores serán llamados a intervenir en el rango que les corresponda en las decisiones de los órganos de poder" (Programa Unidad Popular, 1970: 12-13).

Para Trabajo Social, la estrategia requerida para generar la conciencia crítica y alcanzar el poder popular propuesto en el programa de gobierno, es definida en los siguientes términos:

"El desarrollo de la conciencia pretende la formación de la conciencia crítica a nivel personal, que permita al individuo captar su realidad y la del mundo que lo rodea, pudiendo valorar y enjuiciar esa realidad objetiva y críticamente. Pretende también la formación del sentido de solidaridad y pertenencia a su clase social, que permita al individuo identificarse con ella y participar activamente en las tareas que le son propias. La formación básica sociocultural y política está orientada a la capacitación de los individuos para participar como ciudadanos en la vida del país" (Aylwin, 1970:9).

En el ámbito de la salud pública, el programa de gobierno se proponía "asegurar la atención médica y dental preventiva y curativa para todos los chilenos, financiada por el Estado, los patrones y las instituciones de previsión. Asimismo, se incorporará a la población a la tarea de proteger a la salud pública" (Programa Unidad Popular, 1970:26). Con fecha 01 de septiembre de 1971, se dicta el Decreto 602 del Ministerio de Salud Pública, que crea los Consejos Locales de Salud, a quienes les corresponde "examinar los problemas de salud que afecten a la comunidad; propender a su solución mediante 
acciones rápidas y eficaces; promover el interés de los habitantes para participar en forma activa en la solución de los mismos; colaborar en la divulgación de los planes de acciones de salud, y representar las anomalías que aparezcan en la ejecución de estas acciones" (Art. 3).

En este marco, la participación comunitaria en salud continuaba siendo un eje central en el desarrollo de la estrategia sanitaria y la relación con las organizaciones avanzaba a formas de trabajo de mayor protagonismo social. "En el gobierno de Allende había mucho movimiento de gente, salíamos mucho a trabajar con las distintas organizaciones sociales y también las mismas organizaciones iban al hospital a hacer sus demandas". (Trabajadora Social de Salud. Año de Ingreso al Servicio, 1956).

Las experiencias profesionales profundizaban los esfuerzos de los equipos de salud en torno a la participación de la comunidad en los procesos sanitarios.

Tabla No 4 . Experiencia de participación comunitaria en salud rural

\begin{tabular}{|l|l|}
\hline Nombre experiencia & Formulación de un Programa Integral de Salud Rural \\
\hline Institución & Hospital San Pablo de Coquimbo \\
\hline Equipo de salud & $\begin{array}{l}\text { 4 Médicos Generales de Zona, Enfermera, Matrona, } \\
\text { Asistente Social, Educador Sanitario, Inspector de } \\
\text { Saneamiento, Funcionario de Estadística, Chofer y } 2 \\
\text { Auxiliares de Terreno. }\end{array}$ \\
\hline Período de trabajo & $1969-1971$ \\
\hline \multicolumn{2}{|c|}{ Descripción de la Experiencia } \\
\hline $\begin{array}{l}\text { "Plenamente conscientes de la necesidad de contar con la participación comunita- } \\
\text { ria, nuestro programa centralizó sus acciones hacia la motivación de la comunidad } \\
\text { rural en la búsqueda de soluciones inherentes a sus necesidades sentidas. Uno de } \\
\text { sus problemas básicos era la carencia de recursos para financiar cargos de perso- } \\
\text { nal de colaboración médica en el sector rural; por lo tanto planificamos cursos de } \\
\text { capacitación en salud a nivel de profesores rurales y líderes de la comunidad (...) }\end{array}$ \\
$\begin{array}{l}\text { Estos cursos se han realizado en El Peñón, Tambillos y La Cantera, lugares donde } \\
\text { simultáneamente se realizaban obras de construcción de locales con participación } \\
\text { comunitaria (...) En cuanto al problema relacionado con la comunidad rural, el } \\
\text { programa contempló la prestación de asesoría técnica a las agrupaciones existen- } \\
\text { tes, lo que se tradujo en capacitarlas para lograr su real participación en el proce- } \\
\text { so de desarrollo (...) A través de la labor de asesoría y capacitación de los grupos } \\
\text { existentes, logramos constituir una Junta de Vecinos representativa de la localidad, }\end{array}$ \\
\hline
\end{tabular}


cuya labor estuvo centralizada en abordar problemas de saneamiento básico y lega-
lización de la tenencia de terrenos (...) Simultáneamente, en la localidad de Tongoy
se realizaron acciones de organización de la comunidad, trabajo desempeñado por
una alumna de Servicio Social de la Universidad de Chile de La Serena y supervi-
sada por la profesional del equipo. Se logró la constitución de la Junta de Vecinos
del sector de pescadores y a través de la labor constante de asesoría a la misma y
otras organizaciones existentes, hemos obtenido un nexo vital para el desarrollo
de las acciones de salud contempladas para esa localidad en el Programa de Salud
Integral (...) Aprendí que el desarrollo comunitario se hace cualquier día y a cual-
quier hora”.
Fuente: Aguilera, S. (1971). Ver detalles en bibliografía.

Si bien estas experiencias representaban significativos reconocimientos respecto del aporte de Trabajo Social a la consolidación de la participación social dentro del equipo de salud, la profesión analizaba críticamente las normativas vigentes y exigía un ritmo de cambios de mayor profundidad:

"Las brigadas de voluntarios de salud y los Consejos Locales y/o paritarios de salud, asumen tareas de salud a través de los cuales los pobladores intervienen en el aparato del Estado encargado de atender a las necesidades de salud de la población. Los fines de su intervención son la democratización y la descentralización del servicio; en este sentido los Consejos de Salud han sido concebidos como "gérmenes de poder" en la población, que se abocan al estudio y solución de los problemas de salud. Fueron creados por el Decreto 602, lo que por una parte hace que la democratización del SNS cuente con instrumento legal, pero por otro, limita las atribuciones de los Consejos al subordinarlos a la dirección del Director del Consultorio en el que funcionan. Los Consejos están integrados por representantes de los trabajadores del SNS y de la población, incluyendo CUT y Consejos Campesinos. Algunas limitaciones mostradas en la práctica:

Los integrantes del Consejo Local de Salud son representantes de las organizaciones territoriales, funcionales y laborales de la población, muchas veces de carácter tradicional, con algunos líderes con afanes caudillistas. 
Las tareas que se asignan en el Decreto son esencialmente de carácter asesor y colaborador con el SNS, no constituyendo estructuras de participación en la toma de decisiones a nivel de dirección política, control sobre la programación, manejo de presupuesto y prestación de servicios.

Pero, al mismo tiempo, el Decreto deja margen para que pueda ser utilizado hacia una efectiva movilización popular, la que se desarrolla fundamentalmente al ir enfrentando contingencias, sobre todo cuando tienen carácter de urgencia" (Departamento Poblacional Escuela Trabajo Social Pontificia Universidad Católica de Chile, 1972: 17).

En este marco de planteamientos de mayor compromiso social, se reconoce que la impronta comunitaria enriquece las intervenciones profesionales, al incorporar análisis estructurales y culturales de los problemas de salud que, a la fecha, habían sido tratados históricamente con estrategias asistenciales e individuales. A modo de ilustración, puede señalarse la intervención profesional realizada en el Programa Intracomunitario de Alcoholismo del Servicio Nacional de Salud y dirigido por el Dr. Juan Marconi. Desde este marco institucional y en el contexto de las prácticas profesionales de la escuela de Trabajo Social de la Pontificia Universidad Católica de Chile realizadas en el Servicio Nacional de Salud Área Sur de Santiago, se analizan las características de las estrategias de tratamiento utilizadas entre los años 1957 y 1967. Las prácticas tradicionales se definen como oficialistas, paternalistas y asistencialistas. En ellas "el trabajador social como integrante de los equipos de salud, estaba confinado exclusivamente a la atención del caso social individual, de los enfermos y familiares y un trabajo de grupo en los clubes de alcohólicos recuperados, para fortalecer su organización interna y programar actividades recreativas. Acciones positivas, pero que a la larga por la falta de profesionales que trabajaban en dicho campo y por el exceso de demanda en atención de casos se tornaba improductiva" (Poblete, 1971: 27). Como consecuencia se redefine la intervención profesional, incorporando activamente a la comunidad en el problema. Así, 
entonces, los clubes de abstemios articulados al programa trascienden desde un rol de colaboración pasiva a un rol activo ligado al tratamiento, educación y organización de la comunidad. Lo anterior se traduce en un importante auge de las acciones sanitarias definidas desde la propia comunidad, permitiendo "el aumento notable de clubes de abstemios en el área; la instalación del programa, a instancias de la comunidad, en el Consultorio La Feria de la comuna de San Miguel (350 mil habitantes); y la creación en el Hospital Base Barros Luco-Trudeau, en marzo de 1970, de una consulta externa de alcoholismo y salud mental" (Poblete, 1971: 29). Con la perspectiva del tiempo, esta experiencia ha sido reconocida como la primera experiencia piloto de psiquiatría comunitaria del país, siendo sus componentes claves la capacitación del equipo profesional de atención primaria en el tratamiento del alcoholismo y la promoción de la participación de la comunidad en la solución de sus problemas de salud mental de mayor prevalencia, los que correspondían en conceptos diagnósticos de la época a alcoholismo, neurosis y retraso del desarrollo cognitivo infantil. Esta experiencia piloto a pequeña escala fue diseminada en otros lugares del país, especialmente en el tema de alcoholismo, a través de la atención primaria y grupos de autoayuda (Minoletti y otros, 2012: 441).

Simultáneamente, y a fin de profundizar en la dinámica de la participación social, se inician experiencias de investigación que contextualizan los procesos sanitarios y aportan nuevos referentes culturales para los procesos de trabajo comunitario que se realizaban en contextos urbanos y rurales.

"Fuimos cinco estudiantes de Servicio Social de la sede Valparaíso que fuimos a hacer un trabajo con la Escuela de Antropología de la Universidad de Chile. Queríamos ver las costumbres en relación a la salud que tiene la gente rural. Nos contrató el Servicio Nacional de Salud. Teníamos que hacer una comparación entre la salud urbana y la salud rural. Ver cómo se adaptaban a los cambios. Había que ir con el equipo de salud completo al monte y ver una atención integral de una embarazada o de un anciano. Noso- 
tros veíamos todas las temáticas sociales, cómo curaban sus enfermedades. Se hablaba del mal de ojo, del empacho, de santiguar, o de cómo se ocupaban las hierbas medicinales" (Estudiante Trabajo Social. Período 1967-1971).

Estos valiosos antecedentes, junto con otras experiencias homólogas en diversos puntos del territorio nacional, permitieron fundamentar la propuesta del Programa Sociocultural del SNS, iniciado en 1972 y que propuso una mirada integradora de la salud, asumiendo la multicausalidad de los fenómenos biológicos, e incorporando explícitamente la dimensión cultural a las tareas sanitarias que realizaban a lo largo del país los equipos profesionales y técnicos de la institución.

Los procesos de titulación en Trabajo Social vinculadas a Salud durante 1970-1973, reflejan el compromiso de la profesión con la estrategia de participación comunitaria sanitaria, a través de las siguientes experiencias: La marginalidad como consecuencia del sistema capitalista y la participación social una estrategia para su superación (1971); Democratización del SNS: participación de la comunidad en las tareas de la salud (1972); Mujer y familia en Chile. Diagnóstico y pronóstico para una revolución (1972). Asimismo, las oportunidades profesionales de empleabilidad en el sector salud se mantuvieron en una condición favorable durante el período.

"Yo me demoré días en comenzar a trabajar en salud, porque nosotros egresamos en febrero de 1972, porque la Universidad vivía los días de la Reforma, entonces terminamos en febrero del '72, y yo en abril del '72 estaba trabajando en el Servicio Nacional de Salud. Estaban contratando muchas Asistentes Sociales, específicamente para el área comunitaria" (Trabajadora Social de Salud. Año de Ingreso al Servicio, 1972).

\section{Reflexiones finales}

En ambos períodos presidenciales analizados, las acciones profesionales realizadas en el marco de la participación comunitaria 
en salud contribuyeron de manera decisiva a la redefinición de Trabajo Social desde una concepción predominantemente asistencialista hacia una valorización explícita de la promoción y organización social, redefinición que ha quedado sintetizada en el concepto de agentes de cambio social. Dicho concepto amplificó los desempeños profesionales tradicionales posibles de reconocer en las orientaciones institucionales del sector salud y aportó a un contexto histórico político mayor, caracterizado por cambios estructurales impulsados por el Estado y dirigidos a amplios sectores poblacionales, laborales, sindicales, educacionales, organizacionales y agrarios a lo largo del país que a la fecha sufrían una importante situación de postergación y rezago respecto a los avances en calidad de vida que presentaban sectores de mayores ingresos. Estas acciones profesionales superaron la propia estructura del Servicio Nacional de Salud y avanzaron en el fortalecimiento de una concepción de participación social que a partir de las preocupaciones sanitarias de la época, trascendió en la búsqueda de oportunidades para influir socialmente en la toma de decisiones vinculadas al desarrollo económico, social y cultural del país, posición que encontró favorable acogida en la formación y ejercicio profesional de la época (Castañeda y Salamé 2014: 18).

Sin embargo, los aportes profesionales de Trabajo Social a la participación comunitaria en salud realizados en las etapas gubernamentales revisadas, encontraron un abrupto final a contar del Golpe de Estado de septiembre de 1973. Las acciones impulsadas por el Estado e implementadas por la profesión en torno al ejercicio efectivo de la participación social a través de la promoción popular y del poder popular no contaron con respaldo ideológico para cautelar su proyección en el sistema público de salud en los años dictatoriales, siendo, por el contrario, objetos de censura y represión que ocasionaron el total abandono de las prácticas asociadas y de los avances realizados en esta línea de intervención. Las acciones de salud pública que desarrolló el gobierno militar, apuntaron centralmente a un concepto de salud individual, asistencialista y biologicista, en el marco de una drástica disminución 
de la inversión pública en salud, que encontró su mayor expresión en las reformas estructurales realizadas en la institucionalidad de salud chilena a contar de 1979, que dieron origen a un sistema mixto público-privado, cuyo acento en el acceso y cobertura a la salud conforme el nivel de ingresos individual o familiar, ha desplazado al olvido los esfuerzos solidarios y colectivos que caracterizaron la etapa pre-dictatorial.

Las intervenciones profesionales en salud a partir de septiembre de 1973 se restringen a los ámbitos vinculados a la lógica biomédica, con un marcado énfasis asistencial. Los referentes comunitarios para enfrentar los problemas sanitarios de la población, antes presentes de manera transversal en todas las intervenciones de salud, desaparecen de las estrategias de trabajo.

Tabla No5. Experiencia profesional rehabilitacion en salud

\begin{tabular}{|l|l|}
\hline Nombre experiencia & Tratamiento en Alcoholismo \\
\hline Instituciones & $\begin{array}{l}\text { Programa de Atención del Adulto. Servicio de Salud } \\
\text { Metropolitano Oriente. Región Metropolitana. } \\
\text { Departamento de Psiquiatría y Salud Mental. Univer- } \\
\text { sidad de Chile. }\end{array}$ \\
\hline Equipo de salud & $\begin{array}{l}\text { Médicos Generales, Enfermeras y Asistentes Sociales } \\
\text { con asesoría técnica de la Universidad de Chile. }\end{array}$ \\
\hline Período de trabajo & $1977-1980$ \\
\hline Descripción de la Experiencia \\
\hline $\begin{array}{l}\text { "Este Plan consta de una fase inicial de evaluación médica y psicosocial de los } \\
\text { pacientes nuevos, que es realizada en los consultorios por médicos generales, en- } \\
\text { fermeras y asistentes sociales. Esta fase se acompaña de un esquema de desintoxi- } \\
\text { cación para todos aquellos casos que la requieran. Después de esta fase se motiva } \\
\text { al enfermo para que se incorpore al Club de los Recuperados, donde a través de } \\
\text { grupos se pretende mantener la abstinencia". }\end{array}$ \\
\hline Fuente: Fehuerhake, O. y cols. (1980). Ver detalles en bibliografía. \\
\hline
\end{tabular}

Las nuevas definiciones de los aportes de Trabajo Social en Salud, lo caracterizan como una profesión de perfil funcionario que completa las acciones del nivel primario al cumplir "labores propias de asesoría en todos los programas y de coordinación en diversas instituciones de carácter asistencial y de prevención 
y protección de menores en situación irregular" (France, 1981: 35). En ese marco, el rol profesional fue redefinido desde un perfil marcadamente asistencial, concentrando la tarea en la calificación previsional de los usuarios y en el manejo administrativo de los servicios médicos estadísticos de los establecimientos de salud. Este rol acotado fue acompañado con el congelamiento de las plazas laborales y la desestimación de nuevas contrataciones en los equipos de salud.

"Nuestras principales actividades estaban destinadas a la entrega de la credencial de salud, que era nuestra principal labor en esa época. Tratábamos de realizar intervenciones de otro carácter, más cerca de la comunidad, pero nos presionaban a no realizar ninguna otra acción que no fuese la atención asistencial" (Trabajadora Social. Año de ingreso al Servicio, 1984).

"En ese tiempo estaba perfilado el trabajo en el área salud, más que nada era la entrega de la tarjeta de gratuidad y el equipo desconocía lo que tú hacías. Estar en un consultorio entregando una tarjeta, fue muy cansador y mi rol se remitía sólo a ser una tramitadora. De todas formas, a veces uno forzaba el sistema de manera silenciosa y daba beneficio de gratuidad a personas que lo necesitaban, aunque no calificaran" (Trabajadora Social. Año de ingreso al Servicio, 1986).

Si bien las estrategias de participación social en salud se han retomado en forma progresiva desde el retorno a la democracia hasta la actualidad, y se han traducido en esfuerzos permanentes de los equipos profesionales por incentivar la organización comunitaria, la pérdida de experiencias y continuidad durante 17 años ha opacado los resultados obtenidos en las últimas dos décadas, demostrando que a pesar de los logros que pudiesen haberse alcanzado a la fecha en torno a la resignificación de la participación social en salud, son las condiciones de instrumentalidad y de demanda asistencial de servicios y beneficios las que actualmente prevalecen por sobre la impronta inicial, basada en un concepto de solidaridad y avance mancomunado de la salud pública a partir de los 
esfuerzos compartidos realizados entre la comunidad, los equipos de salud y el Estado.

Como corolario de la absoluta prescindencia de la dimensión comunitaria en el trabajo sanitario en Chile durante la dictadura, destaca el mensaje presidencial del general Augusto Pinochet del 11 de septiembre de 1986, quien haciendo referencia a la salud, la define en esa oportunidad como "estado de completo bienestar físico y psicológico del individuo" (1986: 9), omitiendo abiertamente el componente social, que ha acompañado la clásica definición de la Organización Mundial de la Salud desde el año 1945. Por consecuencia en el período dictatorial, Trabajo Social en su condición de profesión social fue desestimada y cuestionada, debiendo abandonar forzadamente las tareas de participación comunitaria en salud, y redefiniéndose en torno a una posición funcionaria y asistencial de mínimo impacto social, organizada en torno a la atención de individuos, familias y grupos instrumentales convocados a partir de la patología y el daño.

No obstante lo anterior, para la profesión las experiencias de participación social en salud en el período 1960-1973 nutren su memoria colectiva, rescatando los esfuerzos profesionales realizados junto a los equipos de salud de la época, que estuvieron destinados a incorporar a la comunidad en las acciones sanitarias, permitiendo con ello instituir un nuevo rol de promoción social que trascendió la impronta fundacional asistencialista que le caracterizaba hasta ese entonces. Esta búsqueda de un nuevo desempeño profesional se realizó desde el mismo marco institucional de salud que le dio origen en 1925, y que durante el período pre-dictatorial respaldó y legitimó el ejercicio pleno del rol promocional de Trabajo Social en sus tareas sanitarias.

\section{Bibliografía}

Aguilera, S. (1971). Experiencias del equipo de salud del Hospital San Pablo de Coquimbo. Cuadernos Médico Sociales, 4, 32-34. Recuperado el 09.01.2016 de http://cms.colegiomedico.cl/ Magazine/1971/12/4/12_4_8.pdf 
Aylwin, N. (1970). Qué es el trabajo social en la actual perspectiva histórica chilena. Revista de Trabajo Social, 2, 5-10.

Castañeda, P. y Salamé A.M. (2013). Trabajo Social chileno y Gobierno Militar. 40 años de memoria y olvido. Trabajo Social, 84, 55-66.

Castañeda, P. y Salamé A.M. (2014). Trabajo Social chileno y Dictadura Militar. Memoria Profesional predictatorial. Período 1960-1973. Agentes de Cambio y Trauma Profesional. Rumbos TS, 9, 8-25.

Departamento Poblacional. Escuela de Trabajo Social (1972). Algunos elementos del diagnóstico del fenómeno poblacional en Chile. En: Trabajo Social, 7, 7-21.

Fehuerhake, O. y cols. (1980). Investigación, Tratamiento y Prevención del alcoholismo en el área oriente de Santiago. Cuadernos Médico Sociales, 4, 48-52. Disponible en: http:/colegiomedico.cl/lista/volumen/p3//

Figueroa, A. (1979). Evolución del Servicio Social Profesional en Chile durante el período comprendido entre 1925 y 1975. (Memoria de prueba inédita para optar al título de Asistente Social). Universidad de Chile, sede Valparaíso. Departamento de Ciencias Humanas y Desarrollo Social. Escuela de Servicio Social.

France, O. (1981). La Atención Primaria en el Servicio de Salud Metropolitano Oriente. Cuadernos Médico Sociales, 2, 30-35. Recuperado el 09.01.2016 de http://cms.colegiomedico.cl/ Magazine/1981/22/304/22_304_7.pdf

Frei, E. (1964). Esfuerzo de todo un pueblo. Discurso Presidencial para anunciar al país el Programa de Promoción Popular. Santiago de Chile: Partido Demócrata Cristiano. Recuperado el 27.10.2015 de

http://www.archivochile.com/Gobiernos/gob_edo_freim/ de/GOBdefreim0007.pdf

Goic, A. (2015). El sistema de salud en Chile. Una tarea pendiente. Revista Médica de Chile, 143, 774-886. Recuperado el 09.01.2016 de http://www.scielo.cl/pdf/rmc/v143n6/art11.pdf

Gutiérrez A. y Villamar K. (1966). Aspectos que favorecen o dificultan un programa de organización y desarrollo de la comunidad en un área urbana. (Memoria de prueba inédita para optar al título de Asistente Social). Valparaíso: Universidad de Chile, Sede Valparaíso. Facultad de Ciencias Jurídicas y Sociales. Escuela de Servicio Social. 
Instituto Nacional de Estadísticas (1960). Censo Población 1960. Resumen País. Santiago de Chile: INE, Dirección Estadísticas y Censos.

Lavados I. (1984). Evolución de las políticas sociales en Chile. 1964-1980. Santiago de Chile: CEPAL, ILPES, UNICEF.

López T. (2014). El camino recorrido. Intervención comunitaria: cómo es y cómo ha sido la experiencia de los trabajadores sociales chilenos. Santiago de Chile: Librosdementira.

Minoletti, A., Rojas, G. y Horvitz-Lennon, M. (2012). Salud Mental en la Atención Primaria en Chile: aprendizajes para Latinoamérica. En: Cad Saúde Colet, 20, 440-447. Recuperado el 25.11.2015 de http://www.scielo.br/pdf/cadsc/v20n4/ v20n4a06.pdf

Montoya C. y Videla V. (1967). Roles profesionales en el equipo de atención de la salud. Cuadernos Médico Sociales, 2, 13-19. Recuperado el 07.11.2015 de http://cms.colegiomedico.cl/ Magazine/1967/8/2/8_2_6.pdf

Pinochet de la Barra, O. (Ed.) (1992). Eduardo Frei. Obras Escogidas.1931-1982. Colección Chile en el siglo XX. Santiago de Chile: Ediciones del Centro de Estudios Políticos Latinoamericanos Simón Bolívar. Recuperado el 21.11.2015 de http://www.memoriachilena.cl/602/w3-article-19879. html

Poblete, M. (1971). Solución al problema de alcoholismo en manos de una mayoría popular. Trabajo Social 3, 25-30.

Programa Básico de Gobierno de la Unidad Popular (1970). Candidatura Presidencial de Salvador Allende. Recuperado el 10.01.2016 de http://www.memoriachilena.cl/archivos2/ pdfs/MC0000544.pdf

Puentes, R. (1969). Experiencia de un médico general de Zona en Vallenar. Cuadernos Médico Sociales, 3, 32-39. Recuperado el 08.01.2016 de http://cms.colegiomedico.cl/Magazine/1969/10/1/10_1_7.pdf

República de Chile (1967). Decreto 250 del Ministerio de Salud Pública. Crea Consejos Comunitarios de Salud. Santiago de Chile: Ministerio de Salud.

República de Chile (1968). Ley $N^{\circ} 16.880$ de Organizaciones Comunitarias. Santiago de Chile. Recuperado el 25.11.2015 de www. leychile.cl

República de Chile (1971). Decreto 602 del Ministerio de Salud Pública. Crea Consejos Locales de Salud. Santiago de Chile: Ministerio de Salud. 
República de Chile (1986). Mensaje Presidencial. Recuperado el 25.11.2015 de http://www.memoriachilena.cl/602/w3-article-82411.html

Servicio Nacional de Salud (1962). Plan para un Programa de Desarrollo de la Comunidad en la comuna de Conchalí. Santiago de Chile: Servicio Nacional de Salud. (Mimeo).

Sotomayor H. y cols. (1971). Experiencias del equipo de salud del Hospital de Coronel. Cuadernos Médico Sociales, 3, 38-43. Recuperado el 09.01.2016 de http://cms.colegiomedico.cl/Magazine/1971/12/3/12_3_8.pdf

Ugarte J. (1970) Artículo Salud y Comunidad. Cuadernos Médico Sociales 3, 31-32. Recuperado el 11.01.2016 de http://cms.colegiomedico.cl/Magazine/1970/11/3/11_3_8.pdf

Universidad de Chile (1966). Anales de la Facultad de Ciencias Jurídicas y Sociales.1966. Vol. VI Nº 6 (pp. 199-200). Santiago de Chile: Universidad de Chile. Recuperado el 10.01.2016 de https://books.google.cl/books?id=dDzf97L_3SQC\&pg=P A227\&lpg=PA227\&dq=consultorio+maternoinfantil + chi le\&source $=$ bl\&ots $=0$ AkyiKbdJ9\&sig $=$ h7MqO7SjNBp_Pp MEezDFet 1hAJI\&hl=es\&sa $=$ X\&ved=0CBsQ6AEwADgK ahUKEwjHu4TplPDHAhVFQpAKHctoANE\#v=onepage $\& \mathrm{q}=$ consultorio $\% 20$ maternoinfantil $\% 20$ chile $\& \mathrm{f}=$ false

Villalón G. y Vera S. (2012). Panorama Demográfico en Chile contemporáneo. Desafíos para la sociedad del siglo XXI: En: Revista Anales, Séptima Serie, 4, 35-63. Recuperado el 07.01.2016 de www.anales.uchile.cl/index.php/ANUC/article/download/21617/22931 